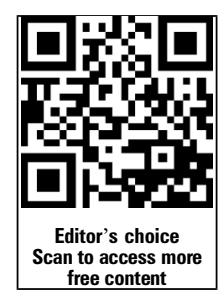
Scan to access mo
free content

\title{
An observational study of shift length, crew familiarity, and occupational injury and illness in emergency medical services workers
}

\author{
Matthew D Weaver, ${ }^{1,2,3}$ P Daniel Patterson, ${ }^{4}$ Anthony Fabio, ${ }^{5}$ Charity G Moore, ${ }^{6}$ \\ Matthew S Freiberg, ${ }^{7}$ Thomas J Songer $^{5}$
}

${ }^{1}$ Department of Emergency Medicine, University of

Pittsburgh, School of Medicine, Pittsburgh, PA, USA

${ }^{2}$ Division of Sleep and Circadian Disorders, Departments of Medicine and Neurology, Brigham and Women's Hospital, Boston, Massachusetts, USA ${ }^{3}$ Division of Sleep Medicine, Department of Medicine, Harvard Medical School, Boston, Massachusetts, USA ${ }^{4}$ Department of Emergency Medicine, Carolinas HealthCare System Medical Center, Charlotte, North Carolina, USA ${ }^{5}$ Department of Epidemiology, University of Pittsburgh, Graduate School of Public Health, Pittsburgh,

Pennsylvania, USA

${ }^{6}$ Dickson Advance Analytics Group, Carolinas HealthCare System, Charlotte, North Carolina, USA

${ }^{7}$ Department of Medicine, Vanderbilt University, School of Medicine, Nashville, Tennessee, USA

\section{Correspondence to}

Matthew D Weaver, Division of Sleep and Circadian Disorders, Departments of Medicine and Neurology, Brigham and Women's Hospital, Landmark Center Suite 301 West, 401 Park Drive Boston, MA 02215 USA; mdweaver@partners.org

Received 25 March 2015 Revised 22 July 2015 Accepted 6 August 2015 Published Online First

14 September 2015

\section{ABSTRACT}

Objectives Emergency medical services (EMS)

clinicians are shift workers deployed in two-person teams. Extended shift duration, workplace fatigue, poor sleep and lack of familiarity with teammates are common in the EMS workforce and may contribute to workplace injury. We sought to examine the relationship between shift length and occupational injury while controlling for relevant shift work and teamwork factors. Methods We obtained 3 years of shift schedules and occupational injury and illness reports were from 14 large EMS agencies. We abstracted shift length and additional scheduling and team characteristics from shift schedules. We matched occupational injury and illness reports to shift records and used hierarchical logistic regression models to test the relationship between shift length and occupational injury and illness while controlling for teammate familiarity.

Results The cohort contained 966082 shifts, 4382 employees and 950 outcome reports. Risk of occupational injury and illness was lower for shifts $\leq 8 \mathrm{~h}$ in duration (RR $0.70 ; 95 \% \mathrm{Cl} 0.51$ to 0.96 ) compared with shifts $>8$ and $\leq 12 \mathrm{~h}$. Relative to shifts $>8$ and $\leq 12 \mathrm{~h}$, risk of injury was $60 \%$ greater (RR 1.60; $95 \% \mathrm{Cl}$ 1.22 to 2.10 ) for employees that worked shifts $>16$ and $\leq 24 \mathrm{~h}$.

Conclusions Shift length is associated with increased risk of occupational injury and illness in this sample of EMS shift workers.

\section{INTRODUCTION}

Emergency medical services (EMS) workers provide the public with medical care and emergent transportation across the USA $24 \mathrm{~h}$ a day. EMS is a high-risk work that involves operating an ambulance at elevated speeds on public roadways, carrying heavy equipment, lifting and moving patients, and stabilising the ill and acutely injured in settings characterised as uncontrolled. ${ }^{1-4}$ Workplace injury among EMS workers is higher than the general working public and other high-risk occupations. ${ }^{5-7}$ EMS workers are commonly deployed in teams of two (a dyad) and work shifts of 12 or 24 h. Recent research raises concern about the safety of EMS workers and patients, revealing a high level of workplace fatigue and limited familiarity between EMS dyadic crewmembers. ${ }^{8} 9$ While fatigue and limited teammate familiarity have been linked to poor safety outcomes and poor performance in a

\section{What this paper adds}

- Occupational injury rates in emergency medical services (EMS) are high, and extended shifts, fatigue and poor sleep quality are common.

- In this sample of nearly one million shifts from 14 EMS agencies, the risk of an occupational injury or illness was found to be associated with increasing shift length.

- This study provides base rate data on EMS shift worker injury, shift duration characteristics, and is the largest US-based study to examine the association between shift duration and risk of work-related injury in the EMS setting.

variety of settings, ${ }^{10}$ there is limited research involving EMS workers and thus lack of evidence to inform investigators and EMS officials with regard to the significance of shift length and crew deployment. $^{8} 11$

The Occupational Safety and Health Administration (OSHA) defines a normal work shift as: "a work period of no more than eight consecutive hours during the day, five days a week with at least an eight-hour period of rest."12 EMS workers are often scheduled to work extended shifts of $\geq 12$ h. Extended shifts increase the risk of adverse events, medical errors and attentional deficits in diverse settings, including healthcare. ${ }^{13}$ Recent research using cross-sectional survey data links EMS workplace injury to fatigue. ${ }^{9}$ Shift length was not a factor in this study, yet there is ongoing uncertainty and debate on the contribution and significance of shift length, timing and rotation in EMS workplace safety. ${ }^{11}$ Specifically, many in EMS administration perceive extended shifts as dangerous and advocate they be eliminated. ${ }^{14}$ Others, including EMS workers, may feel differently given a lack of research showing a direct link between shift work factors, including length, and safety outcomes, such as injury or medical error.

Of equal importance is EMS crew deployment and its association with safety. There is an emerging body of literature that raises concern for the lack of planned deployment of EMS crews. Most EMS systems staff two crewmembers on each ambulance. One recent study shows that EMS workers are 
scheduled with 19 different crewmembers annually, with some working with more than 50 different partners in 1 year. ${ }^{8}$ Lack of familiarity between pilots and co-pilot crews has been linked to a higher rate of errors during take-off and landing versus pilot/co-pilot teams with greater teammate familiarity. Another analysis of aviation data shows that greater than $70 \%$ of accidents can be traced back to the pilot/co-pilot crew's first flight first day together. ${ }^{15}$ Other studies also link limited familiarity between teammates with poor performance and poor outcomes. ${ }^{16}{ }^{17}$ Because EMS work depends on two crewmembers working well together under stressful conditions, there is reason to believe that the dangers of extended shift work combined with limited teammate familiarity pose a risk to safety.

In this paper, we explore the role extended shifts and teammate familiarity may play in EMS worker injury and illness. The purpose of this analysis was to evaluate the impact of shift length on internal reports of occupational injury and illness in a national cohort of EMS workers. We hypothesised that the risk of injury or illness would increase with increasing shift length.

\section{METHODS}

This is an analysis of administrative data from a retrospective cohort of 14 EMS agencies with 37 base sites. The exposure of interest was shift length. The outcome of interest was OSHA reported injury or illness on the shift under study. Participating organisations represent a convenience sample of organisations providing historical scheduling records and occupational injury records for a period of $1-3$ years.

\section{Study protocol}

Agencies provided historical employee shift schedules and OSHA occupational injury or illness reports. OSHA reports were matched to specific shifts using a combination of variables including date, location (agency/base site) and employee identification number. In cases where the employee and location matched the OSHA report, but the shift start date did not, the OSHA report was matched to the most proximal previous work shift as long as the shift occurred within 4 days of the recorded injury or illness. The 4-day interval was used to maximise the likelihood that the OSHA report be matched to the shift on which the incident occurred. OSHA stipulates an injury must be recorded within 7 days. Shifts were excluded when the assigned job role described a non-clinical task, for example, maintenance worker, billing staff or vehicle service technician.

\section{Outcome variable of interest}

The outcome of interest was work-related injury or illness recorded on the agency OSHA form $300 \log { }^{18}$ The OSHA form $300 \log$ from each of the participating EMS agencies was obtained for all 37 base sites. The OSHA form contains where the event occurred, a short, free-text description of the event along with the assignment of the event into categories of injury or illness, with several subcategories beneath the illness designation. The assignment of the event into categories is performed by an individual at the EMS agency. The outcome was defined as any report from the OSHA log provided it met the following criteria: injury - any wound or damage to the body resulting from an event in the work environment, requiring medical treatment beyond basic first aid, or resulting in loss of consciousness or an inability to perform normal duties without restriction. Illness or unanticipated exposure to illness was defined as any illness or exposure to infectious illness that resulted from an event in the work environment and was not prevented by the use of the personal protective equipment. Routine patient care of infectious persons in the course of duty without incident did not meet the threshold for a report and was excluded as an outcome. Each reported injury or illness was reviewed to determine whether or not the report met the criteria for inclusion as an outcome. The purpose of reviewing each record for inclusion was to minimise potential biases that could be present in cases of differential thresholds for reporting injuries or illnesses across agencies and individuals.

We grouped the injury locations into common categories. Locations included the emergency scene, in the ambulance or during transport, the hospital facility and the agency home base site. We then searched the narrative description of the event to estimate the prevalence of keywords specific to types of injuries, actions and bodily regions.

\section{Exposure of interest}

The primary independent variable of interest was the length of the shift. Shift length was extracted from historical shift schedules. Shift length was treated in several ways for completeness. First, EMS agencies commonly schedule shifts of $8,12,16$ and $24 \mathrm{~h}$. The primary representation of the exposure in this analysis stratifies the continuous shift hours variable into these four sections: shifts $\leq 8 \mathrm{~h}$ were grouped together, shifts $>8$ and $\leq 12 \mathrm{~h}$, then shifts $>12$ and $\leq 16 \mathrm{~h}$, shifts $>16$ and $\leq 24 \mathrm{~h}$, and shifts $>24 \mathrm{~h}$. Next, shift length was examined as a dichotomous measure, considering shifts $\geq 12 \mathrm{~h}$ to be extended in nature. We also performed a similar test considering shifts $\geq 10 \mathrm{~h}$ as exposed to extended work hours. Shift length was also classified into categories of $8,12,16$ and $24 \mathrm{~h}$. The categories were constructed by assigning shifts $\leq 10 \mathrm{~h}$ in the $8 \mathrm{~h}$ category, shifts $>10$ and $\leq 14 \mathrm{~h}$ in the $12 \mathrm{~h}$ category, shifts $>14$ and $\leq 18 \mathrm{~h}$ in the $16 \mathrm{~h}$ category, and all shifts $>18 \mathrm{~h}$ in the $24 \mathrm{~h}$ category. Lastly, shift length was treated as a continuous variable.

We present two approaches to classify the exposure of shift length. In the a priori approach, the shifts were treated exactly as listed on the schedule. In situations where an employee was scheduled to work two $8 \mathrm{~h}$ shifts without rest with two different partners, these shifts were analysed as two separate $8 \mathrm{~h}$ shifts to allow for control of familiarity between the two partners. We then performed a practical analytic approach designed with the intent of estimating the cumulative hours of work providers were scheduled for, regardless of transitions between partners. In the practical approach, shifts beginning $\leq 1 \mathrm{~h}$ after the end of the most recent shift were combined and treated as a single, continuous shift.

\section{Independent variables of interest}

Teammate familiarity was defined as the number of shifts the employee worked with the partner(s) assigned for the shift of the interest within the 8 weeks preceding the shift. The familiarity variable was categorised using quartiles. The 8 -week interval was chosen based on prior literature suggesting that 8 weeks is the maximum period of recall of team interactions. ${ }^{19} 20$

Other relevant covariates were also constructed from the shift schedules. The recovery period was calculated as the elapsed time between the end of the most recent shift and the beginning of the next shift. Recovery was treated as a continuous variable in $1 \mathrm{~h}$ increments. Each shift was classified as overnight or not. The following were defined as overnight shifts: shifts of at least $16 \mathrm{~h}$ duration starting at or after 15:00, shifts of at least $12 \mathrm{~h}$ duration starting at or after 18:00, shifts of at least $8 \mathrm{~h}$ starting at or after 22:00, and all shifts lasting $24 \mathrm{~h}$ or more. Any shift with a start time $\leq 2 \mathrm{~h}$ after the end of the most recent shift was considered consecutive in nature. 
Part-time employees were defined consistent with the Bureau of Labor Statistics (BLS) standard. ${ }^{21}$ The BLS considers an employee to be part-time if their hours do not exceed $34 \mathrm{~h} /$ week. Part-time status was determined by calculating retrospectively the average work hours for that employee in the 4 weeks immediately preceding each shift. Thus, an employee could transition from part-time to full-time status depending on their work schedule throughout the study period.

Workforce size has been associated with injury reporting in other settings. ${ }^{22} 23$ The number of unique employees working a shift during a 4-week period was used to estimate the number of workers employed by each agency. The month corresponding to the midpoint of the data collection period was isolated to generate this estimate.

\section{Statistical analysis}

The variables of interest are reported using mean and SD when normality is present, and with median and IQR otherwise. The rate of OSHA reports was calculated as the number of reports per 100 full-time equivalent (FTE). An FTE was defined as $2000 \mathrm{~h}$ of work per year.

Multivariable mixed-effects logistic models were constructed for hypothesis testing. The variables of interest were specified a priori. In the a priori approach, the fixed effects were the length of the shift in hours, categorical quartile of familiarity of crew on the shift, hours of recovery, whether or not the shift encompassed overnight hours, whether the shift started within $2 \mathrm{~h}$ of the most recent shift end (consecutive shift) full-time versus part-time employment status, and the estimated size of agency's workforce. A random agency effect was utilised to account for the clustering of EMS workers within agencies and a random worker effect was implemented to account for the correlation between repeated measures within worker. The practical approach was identical to the a priori approach, except teammate familiarity was not included in the modelling strategy. The analysis was performed using Stata V.12.1 MP, College Station, Texas, USA: StataCorp LP.

The primary outcome was the presence/absence of an OSHA reportable occupational injury or illness. A sensitivity analysis was performed to examine the association excluding reports classified as illness as they are often exposures to infectious illness, and may have a different relationship with shift length compared with that of injury.

\section{Ethical statement}

This study was reviewed and approved by the University of Pittsburgh Institutional Review Board.

\section{RESULTS}

\section{Study sample}

Administrative shift scheduling and injury data were obtained from 14 EMS organisations (table 1). Half of the agencies were located in the West USA census region $(n=7)$. Four agencies were located in the South, another two agencies were in the Northeast, and one agency serviced the Midwest census region.

We obtained 989444 shifts and 972 OSHA reports. After removal of non-clinical and incomplete shift records, 966082 total work shifts from 4382 employees were available for analysis. Twelve OSHA reports did not meet the criteria for reporting and were excluded from the analysis.

\section{Shift length}

The mean shift length overall was $12.6 \mathrm{~h}$ (SD 4.40), while the median length was $12 \mathrm{~h}$ (IQR 12, 12; table 1$)$. The average shift length varied across agencies $(\mathrm{p}<0.001)$. The minimum agency median was $8 \mathrm{~h}$ (IQR 8-8), while the maximum agency median was $24 \mathrm{~h}$ (IQR 12-24).

\section{Injury or illness}

The overall rate of OSHA reports in this sample was 15.59/100/ year (table 1). There were a total of 950 reports from 677 employees. Nearly $75 \%$ of reports were categorised as injuries (table 2). One in five injuries or illnesses resulted in the individual being restricted in their normal work activities (22.3\%), while $12.7 \%$ resulted in time away from work.

The location of the injury or illness was reported for 878 reports $(92.4 \%)$. The emergency scene was the most common location for injuries and illnesses to occur $(n=454,51.7 \%)$. Another 29.2\% occurred in the ambulance or during transport $(n=256)$. Approximately one in six occurred at the receiving

Table 1 Agency workforce, scheduling characteristics and occupational injury and illness rates

\begin{tabular}{|c|c|c|c|c|c|c|c|c|}
\hline Location & Number of employees & $\begin{array}{l}\text { Proportion part-time } \\
\text { employees (\%) }\end{array}$ & Start date & End date & $\begin{array}{l}\text { Number of } \\
\text { shifts ( }(\%))\end{array}$ & $\begin{array}{l}\text { Shift length } \\
\text { (median, IQR) }\end{array}$ & OSHA reports & $\begin{array}{l}\text { Rate } \\
\text { (per } 100 \text { FTE) }\end{array}$ \\
\hline Agency 1 & 545 & 30.17 & $1 / 1 / 11$ & $6 / 30 / 13$ & 143119 (14.81) & $12(12-12)$ & 90 & 10.15 \\
\hline Agency 2 & 153 & 58.33 & $11 / 1 / 12$ & $11 / 29 / 13$ & 19190 (1.99) & $12(12-12)$ & 23 & 20.93 \\
\hline Agency 3 & 231 & 68.24 & $1 / 1 / 11$ & $6 / 30 / 13$ & $65229(6.75)$ & $12(12-12)$ & 26 & 6.68 \\
\hline Agency 4 & 254 & 37.24 & $2 / 27 / 11$ & $11 / 29 / 13$ & $50237(5.20)$ & $12(12-24)$ & 68 & 16.58 \\
\hline Agency 5 & 170 & 14.53 & $3 / 27 / 11$ & $11 / 29 / 13$ & 42006 (4.25) & $24(12-24)$ & 20 & 4.81 \\
\hline Agency 6 & 491 & 42.75 & $1 / 1 / 11$ & $11 / 29 / 13$ & $131182(13.58)$ & $12(12-12)$ & 136 & 16.99 \\
\hline Agency 7 & 314 & 41.40 & $1 / 1 / 11$ & $11 / 29 / 13$ & 93661 (9.69) & $12(12-12)$ & 51 & 9.29 \\
\hline Agency 8 & 220 & 61.46 & $5 / 21 / 11$ & $11 / 29 / 13$ & $41061(4.25)$ & $12(9-12)$ & 40 & 17.32 \\
\hline Agency 9 & 387 & 78.04 & $10 / 1 / 11$ & 9/30/13 & $61751(6.39)$ & $12(12-12)$ & 219 & 56.37 \\
\hline Agency 10 & 262 & 53.38 & $1 / 1 / 11$ & $11 / 29 / 13$ & $58247(6.03)$ & $12(11-13)$ & 36 & 10.02 \\
\hline Agency 11 & 447 & 48.64 & $1 / 22 / 11$ & $11 / 29 / 13$ & $94290(9.76)$ & $12(10-12)$ & 50 & 8.48 \\
\hline Agency 12 & 516 & 55.41 & $1 / 1 / 11$ & $11 / 29 / 13$ & $90988(9.42)$ & $12(9-12)$ & 23 & 4.53 \\
\hline Agency 13 & 154 & 94.20 & $10 / 31 / 11$ & $8 / 26 / 13$ & $48041(4.97)$ & $8(8-8)$ & 156 & 81.18 \\
\hline Agency 14 & 241 & 64.94 & $10 / 1 / 11$ & $7 / 31 / 13$ & $27080(2.80)$ & $24(12-24)$ & 12 & 4.57 \\
\hline Total & 4382 & 52.38 & $1 / 1 / 11$ & $11 / 29 / 13$ & $966082(100)$ & $12(12-12)$ & 950 & 15.59 \\
\hline
\end{tabular}

OSHA, Occupational Safety and Health Administration. 
Table 2 A summary of the work-related injuries and illnesses reported during the study period and their severity

\begin{tabular}{|c|c|c|}
\hline & $\begin{array}{l}\text { Total reports } \\
(n=950,100 \%)\end{array}$ & $\begin{array}{l}\text { Median } \\
\text { (p25-p75) }\end{array}$ \\
\hline \multicolumn{3}{|l|}{ Category } \\
\hline Injury & $705(74.2)$ & - \\
\hline Illness & $245(25.8)$ & - \\
\hline Skin disease or disorder & $4(0.4)$ & - \\
\hline Respiratory condition & $16(1.7)$ & - \\
\hline Poisoning & $2(0.2)$ & - \\
\hline Hearing loss & $3(0.3)$ & - \\
\hline All other illnesses & $169(17.8)$ & - \\
\hline Not specified & $51(5.4)$ & - \\
\hline \multicolumn{3}{|l|}{ Severity } \\
\hline Restricted work activity & $212(22.3)$ & $8(1-23)$ \\
\hline Days away from work & $121(12.7)$ & $8(2-25)$ \\
\hline Death & $0(0.0)$ & - \\
\hline Other or unspecified & $617(64.9)$ & - \\
\hline
\end{tabular}

facility $(n=135,15.4 \%)$, and 33 reports originated from an event at the base site itself (3.8\%).

All reports were accompanied by a short description of the event. Among the 705 injury reports, the words 'sprain' or 'strain' were present in over three-fourths of descriptions $(\mathrm{n}=558,79.2 \%)$. Contusions or abrasions were reported in 84 descriptions (11.9\%). There were 21 events described as concussions (3.0\%) and 15 descriptions of needle stick injuries (2.1\%). The words 'lifting', 'moving' or 'transferring' were listed for nearly half of all injuries $(n=345,48.9 \%)$ and the most commonly listed body part was the neck or back $(n=321,45.5 \%)$.

\section{Independent variables}

Teammate familiarity was greatest for shifts $>12$ and $\leq 16$ h duration, where partners had a median of 11 shifts together in the previous 8 weeks (table 3). Consecutive shifts were most common for longer shift length categories. Over 20\% of shifts longer than $24 \mathrm{~h}$ began immediately following the end of the most recent previous shift. Hours of recovery were greatest for shifts $16-24 \mathrm{~h}$, with a median of 2 days off-work prior to those shifts in this cohort. Shifts $8-12 \mathrm{~h}$ in duration had a median of $12 \mathrm{~h}$ recovery. Although shifts longer than $24 \mathrm{~h}$ were rare, over
$15 \%$ of all workers in this sample worked at least one shift of this type during the study period.

\section{Univariable models}

Shift length was associated with reported injury or illness without adjustment for confounding variables $(p=0.003)$, while teammate familiarity was not $(p=0.62)$. A quadratic term for shift length was not significant, suggesting a linear relationship between shift length and the outcome. Other covariates of interest, including overnight shift, consecutive shift, hours of recovery, part-time worker status and agency workforce size were not associated with the outcome.

\section{A priori approach: multivariable models}

Compared with shifts $>8$ and $\leq 12 \mathrm{~h}$, shifts $>12$ and $\leq 16 \mathrm{~h}$ increased the risk by $27 \%$ (this increase was not statistically significant). Shifts greater than $16 \mathrm{~h}$ and as long as $24 \mathrm{~h}$ increased the risk of injury or illness by $60 \%$. Shifts $\leq 8 \mathrm{~h}$ in duration decreased the risk of occupational injury or illness by $30 \%$ (table 4). Characterising shift length in other ways yielded similar results. Shifts $12 \mathrm{~h}$ in duration or greater increased the risk of occupational injury or illness by $49 \%$ (RR 1.49 ; $95 \%$ CI 1.18 to 1.88$)$. For every additional hour of shift length, the risk of injury or illness increased by 4\% (RR 1.04; 95\% CI 1.02 to 1.06; table 5).

Familiarity, agency workforce size, part-time status and hours of recovery were not associated with occupational injury or illness. Consecutive shifts also did not significantly alter the risk of occupational injury or illness. Overnight shifts were safer, demonstrating a $22 \%$ decrease in risk compared with all other shifts (table 4).

\section{Practical approach: multivariable models}

An alternative analysis of the data where shifts with $\leq 1 \mathrm{~h}$ recovery time were combined found that longer shifts remained associated with significantly increased risk of injury or illness. Relative to shifts of $\leq 8 \mathrm{~h}$ duration, shifts $>12$ and $\leq 16 \mathrm{~h}$ increased risk by $43 \%$, while shifts $>16$ and $\leq 24$ h increased risk by $93 \%$. Shifts lasting longer than $24 \mathrm{~h}$ had nearly a threefold increase in risk (table 5). Shifts $\geq 12 \mathrm{~h}$ conferred a $38 \%$ increased risk of injury or illness.

\section{DISCUSSION}

The analysis suggests an increased risk of injury and illness with increased shift duration. The effect was statistically significant

Table 3 Shift characteristics across duration of shift categories

\begin{tabular}{|c|c|c|c|c|c|c|}
\hline & $\begin{array}{l}\leq 8 h \\
(n=121093)\end{array}$ & $\begin{array}{l}>8 \text { and } \leq 12 h \\
(n=675630)\end{array}$ & $\begin{array}{l}>12 \text { and } \leq 16 h \\
(n=61239)\end{array}$ & $\begin{array}{l}>16 \text { and } \geq 24 h \\
(n=106267)\end{array}$ & $\begin{array}{l}>24 h \\
(n=1853)\end{array}$ & $\begin{array}{l}\text { Total } \\
(n=966082)\end{array}$ \\
\hline Overnight shifts (n, \%) & $15237(12.58)$ & $156420(23.15)$ & $4900(8.00)$ & $100296(94.38)$ & $1853(100)$ & $101949(10.55)$ \\
\hline Partner familiarity (median, IQR) & $3(0,13)$ & $8(1,21)$ & $11(1,20)$ & $4(0,11)$ & $6(2,13)$ & $7(1,19)$ \\
\hline Consecutive shifts (n, \%) & $10791(8.91)$ & $14108(2.09)$ & $899(1.47)$ & $10906(10.26)$ & $385(20.78)$ & 37089 (3.84) \\
\hline Hours of recovery (median, IQR) & $16(16,46)$ & $12(12,60)$ & $24(11-59)$ & $48(24,73)$ & $24(7.5,60)$ & $16(12,60)$ \\
\hline $\begin{array}{l}\text { Number of shifts past } 7 \text { days } \\
\text { (median, IQR) }\end{array}$ & $4(3,5)$ & $3(3,4)$ & $3(2,4)$ & $2(2,3)$ & $3(2,4)$ & $3(2,4)$ \\
\hline Unique workers $(n, \%)^{*}$ & $3490(79.64)$ & $4141(94.50)$ & $2144(48.93)$ & $2017(46.03)$ & $681(15.54)$ & $4382(100)$ \\
\hline Total injuries and illnesses (n, \%) & $197(0.16)$ & $586(0.09)$ & $49(0.08)$ & $117(0.11)$ & $1(0.05)$ & $950(0.10)$ \\
\hline Resulting in work restriction & $12(0.01)$ & $146(0.02)$ & $13(0.02)$ & $40(0.04)$ & $1(0.05)$ & $212(0.02)$ \\
\hline $\begin{array}{l}\text { Resulting in time away from } \\
\text { work }\end{array}$ & $43(0.04)$ & $56(0.01)$ & $6(0.01)$ & $16(0.02)$ & $0(0.00)$ & $121(0.01)$ \\
\hline
\end{tabular}


Table 4 A priori approach: multivariable model results for predicting injury as a function of shift length and other characteristics

\begin{tabular}{|c|c|c|}
\hline & Relative risk (95\% Cl) & p Value \\
\hline \multicolumn{3}{|l|}{ Shift length (category) } \\
\hline$\leq 8 \mathrm{~h}$ & 0.70 (0.51 to 0.96$)$ & 0.029 \\
\hline$>8$ and $\leq 12 \mathrm{~h}$ & Referent & - \\
\hline$>12$ and $\leq 16 \mathrm{~h}$ & $1.27(0.91$ to 1.77$)$ & 0.158 \\
\hline$>16$ and $\leq 24 \mathrm{~h}$ & $1.60(1.22$ to 2.10$)$ & 0.001 \\
\hline$>24 \mathrm{~h}$ & $1.18(0.16$ to 8.49$)$ & 0.87 \\
\hline Overnight shift & 0.78 (0.65 to 0.93$)$ & 0.005 \\
\hline Consecutive shift & $0.84(0.62$ to 1.15$)$ & 0.29 \\
\hline \multicolumn{3}{|l|}{ Crew familiarity } \\
\hline $0-1$ shifts & Referent & - \\
\hline $2-7$ shifts & 1.05 (0.88 to 1.24$)$ & 0.59 \\
\hline 8-19 shifts & $1.08(0.90$ to 1.30$)$ & 0.39 \\
\hline$\geq 20$ shifts & $1.14(0.91$ to 1.41$)$ & 0.25 \\
\hline Hours of recovery & $1.00(1.00$ to 1.00$)$ & 0.85 \\
\hline \multicolumn{3}{|l|}{ Employment status } \\
\hline Full-time & Referent & - \\
\hline Part-time & $0.91(0.78$ to 1.07$)$ & 0.26 \\
\hline Midpoint agency size (20 person units) & 0.99 (0.86 to 1.13$)$ & 0.88 \\
\hline
\end{tabular}

for $12 \mathrm{~h}$ shifts compared with $<12 \mathrm{~h}$ shifts and the greatest risk was observed for $24 \mathrm{~h}$ shifts. Shift length has a linear relationship with occupational injury and illness in this sample.

The relationship between shift length and safety outcomes is poorly understood and little data exist to guide decisionmaking. ${ }^{11}$ The endotracheal intubation success rates of Air Medical providers was evaluated at one organisation after a change in scheduling policies. ${ }^{24}$ Success rates did not vary by increasing shift length from 12 to $24 \mathrm{~h}$. Thomas et al ${ }^{25}$ similarly found no difference in cognitive performance for 12 vs $18 \mathrm{~h}$ shifts in a population of 10 flight nurses. Another effort in Air Medical providers found no difference in cognitive performance between 12 and $24 \mathrm{~h}$ shifts, and also reported reduced fatigue at the end of a $24 \mathrm{~h}$ shift compared with the beginning. ${ }^{26} \mathrm{~A}$ crosssectional survey of 511 EMS providers nationally found the proportion of severely fatigued providers was highest among those working $24 \mathrm{~h}$ shifts, and that severe mental or physical fatigue was associated with injury, medical errors and safetycompromising behaviours. Shift length was associated with these outcomes on univariate analysis, but not after fatigue was also included in the model. ${ }^{9}$

The rate of fatal injuries in EMS workers exceeds that of the general public, and transportation crashes are the most common cause. ${ }^{5}$ Driving emergently relies on reaction time and judgement for safety, both of which are impaired by fatigue. ${ }^{28} 29$ Drowsiness increases the risk of a crash eightfold. ${ }^{30}$ Multiple studies have demonstrated that the degree of impairment with sustained wakefulness is similar to alcohol intoxication. ${ }^{31}$ Individuals who are sleep-deprived also have difficulty processing information and adapting to changing circumstances-tasks that are critical for the safety of EMS workers. ${ }^{32}$ Our data suggest that EMS workers may not arrive to work fully restedobtaining only $6 \mathrm{~h}$ of preshift sleep on average, ${ }^{33}$ and shifts of $12 \mathrm{~h}$ or more are most common. These factors combined suggest that without restorative rest, impaired mental and physical performance may be present.

EMS agencies vary widely in terms of structure, coverage area, demands for service, monetary resources, available workforce and other factors. There is no optimal schedule to meet the needs of all potential workplaces. In many cases, adequate staffing would not be possible without extended shifts. Extended shifts that allow for restorative sleep and rest may protect against the development of fatigue and sleepiness. Frakes and Kelly ${ }^{34}$ showed EMS providers averaged $7 \mathrm{~h}$ of sleep on $24 \mathrm{~h}$ shifts, and Guyette et $a l^{26}$ observed improved performance on select tasks at the end of a $24 \mathrm{~h}$ shift compared with the beginning, likely aided by on-shift sleep. Studies of innovative scheduling practices among EMS providers in Japan suggest

Table 5 A priori versus practical approach: multivariable estimates of risk describing the association between shift length and occupational injury or illness depend on the definition of exposure

\begin{tabular}{|c|c|c|c|c|}
\hline & \multicolumn{2}{|l|}{$\begin{array}{l}\text { A priori approach } \\
\mathrm{N}=961827\end{array}$} & \multicolumn{2}{|l|}{$\begin{array}{l}\text { Practical approach } \\
\mathrm{N}=926763\end{array}$} \\
\hline & Relative risk (95\% Cl) & p Value & Relative risk $(95 \% \mathrm{Cl})$ & $\mathrm{p}$ Value \\
\hline$\leq 8 \mathrm{~h}$ & Referent & - & Referent & - \\
\hline$>8$ and $\leq 12 \mathrm{~h}$ & 1.43 (1.04 to 1.97$)$ & 0.03 & 1.16 (0.88 to 1.53$)$ & 0.30 \\
\hline$>12$ and $\leq 16 \mathrm{~h}$ & 1.82 (1.17 to 2.82$)$ & 0.008 & $1.43(1.05$ to 1.95$)$ & 0.025 \\
\hline$>16$ and $\leq 24 \mathrm{~h}$ & 2.29 (1.52 to 3.46$)$ & $<0.001$ & 1.93 (1.33 to 2.81$)$ & 0.001 \\
\hline$>24 \mathrm{~h}$ & $1.68(0.23$ to 12.42$)$ & 0.61 & 2.88 (1.74 to 4.77$)$ & $<0.001$ \\
\hline Continuous (hours) & 1.04 (1.02 to 1.07$)$ & $<0.001$ & 1.03 (1.02 to 1.04$)$ & $<0.001$ \\
\hline$\geq 10 \mathrm{~h}$ & 1.37 (1.05 to 1.77$)$ & 0.018 & 1.24 (0.99 to 1.56$)$ & 0.06 \\
\hline$\geq 12 \mathrm{~h}$ & 1.49 (1.18 to 1.88$)$ & 0.001 & $1.38(1.12$ to 1.70$)$ & 0.002 \\
\hline $8 \mathrm{~h}$ category & Referent & - & Referent & - \\
\hline $12 \mathrm{~h}$ category & $1.33(1.05$ to 1.70$)$ & 0.018 & 1.22 (0.96 to 1.54$)$ & 0.10 \\
\hline $16 \mathrm{~h}$ category & 2.08 (1.12 to 3.86$)$ & 0.021 & 1.42 (0.99 to 2.05$)$ & 0.06 \\
\hline $24 \mathrm{~h}$ category & 2.05 (1.44 to 2.90$)$ & $<0.001$ & 2.13 (1.54 to 2.96$)$ & $<0.001$ \\
\hline
\end{tabular}

Each estimate in the table represents a separate multivariable model adjusted for the previously described covariates.

Estimates between the lines are from different models. 
that protected intershift rest periods may alleviate perceived fatigue. ${ }^{35}$ However, some workplaces do not permit sleep while employees are on shift, and calls for service may preclude rest opportunities.

Trials of novel, minimally intrusive, intrashift and intershift safety management interventions in the EMS setting are needed. One intrashift intervention used text messages to perform momentary assessments of EMS workers while they were on-shift. Interventional messages were sent if employees reported severe fatigue, sleepiness or difficulty with concentration. ${ }^{36}$ Participants were highly compliant, and the intervention reduced fatigue and sleepiness in a subgroup of participants.

The evidence provided by this study should be viewed with caution if considering a change in policy. These data have important limitations and depending on how the exposure is treated (table 5), different conclusions can be drawn regarding the safety of given shift length. Despite this, these data show a consistent message. The findings are early observational evidence of a preventable exposure associated with injury and illness and should be tested further in a randomised design.

\section{Limitations}

There are several important limitations that should be considered when interpreting our results. First, this is an observational study, and neither the exposure nor the outcome was recorded with the intent to examine the hypothesised question. Minimal agency-level demographic information was collected. Based on the number of active employees at each agency during the study period, our sample is not representative of agencies with workforces with less than 100 employees. There is no information on call volume, rurality or existing fatigue management systems, important factors when interpreting these results. It is possible that this sample is composed entirely of high volume non-rural agencies, where rest on shift may be uncommon or not permitted. We observed a protective effect for night shifts in this analysis, potentially because call volume is often lower on night shifts, offering decreased exposure to occupational hazards. ${ }^{32}$ Call volume could be an important driver of the associations seen in this analysis and future research should seek to capture this information. Extended duration shifts with low call volume may allow for rest on-shift. We do not know the workload of the crew for any shift length in this analysis.

The social norms at participating agencies regarding injury reporting are unknown. Safety culture has been associated with injury reporting in other settings, with a higher rate of underreporting in workplaces with negative safety culture. ${ }^{37}$ In EMS, previous research suggests higher odds of self-reported occupational injury in agencies with negative safety culture. ${ }^{38}$ Safety culture was not available as a covariate in this analysis. We attempted to minimise biases from agencies who recorded minor events by reviewing each outcome to determine if it met the threshold for reporting. Twelve reports did not meet the outcome definition and were excluded.

Relevant individual characteristics, such as age, sex, medical conditions and personal lifestyle habits, were not available. The sleep habits of individuals in the study could potentially explain our findings. The number of jobs that each individual worked was not known. Many EMS workers are employed at multiple agencies simultaneously, ${ }^{93}$ and the likelihood of employment at more than one agency may be related to the most common shift length worked. Individuals who primarily work $24 \mathrm{~h}$ shifts may have greater time availability with which to obtain other employment. Multiple job holders may be at increased risk of fatigue and injury due to a combination of factors, including reduced sleep, increased fatigue, long work hours and increased commuting time. ${ }^{39}$

The use of OSHA 300 logs to capture the outcome has limitations. OHSA logs are widely believed to underestimate the burden of injury and illness, somewhere between $20 \%$ and $70 \% .{ }^{40}{ }^{41}$ Under-reporting may be especially prevalent among healthcare workers. ${ }^{18}$ Evidence suggests under-reporting is particularly common in instances of musculoskeletal injury and needlestick injury, among the most common injuries sustained by EMS providers. ${ }^{5}$ We grouped reports of injuries and illness together in our analysis. Examining only the outcomes recorded as injuries yielded similar results. We used keywords within the narrative to estimate prevalence of injury characteristics. This approach may not capture all injuries within a category of interest, and not all possible keywords were searched. Any events related to shift length that occurred outside of work hours were not captured. There is an increased risk of motor vehicle crash commuting to and from the workplace for extended shifts, with a $9 \%$ increased crash risk for every $24 \mathrm{~h}$ or longer shift. ${ }^{42}$

The data set lacked granularity to examine the evolution of risk over successive hours on duty. The OSHA report was matched to a shift, with no knowledge of how many hours into the shift the injury or illness occurred. OSHA reports were also matched to the most recent shift within the previous 4 days. This method assumed that the event described occurred on the most recent shift. If reporting was delayed or inaccurate, the report may not be matched to the shift on which it occurred.

\section{CONCLUSION}

Extended shift duration is associated with occupational injury and illness among EMS shift workers. We believe randomised controlled trials of diverse agency scheduling practices, intrashift and intershift interventions are needed.

Acknowledgements The authors acknowledge the University of Pittsburgh, Department of Emergency Medicine, where the work was performed.

Contributors MDW and PDP conceived the study and acquired the data. All authors substantially contributed to the design, analysis and interpretation of the study. MDW drafted the manuscript and all authors contributed substantially to its revision. All authors gave final approval of the version to be published.

Funding Work performed on this study was supported by a grant from the Centers for Disease Control and Prevention (CDC), National Institute for Occupational Safety and Health (NIOSH), Grant \#1R210H010025-01A1.

Competing interests None declared.

Provenance and peer review Not commissioned; externally peer reviewed.

\section{REFERENCES}

1 Corbett SW, Grange JT, Thomas TL. Exposure of prehospital care providers to violence. Prehosp Emerg Care 1998;2:127-31.

2 Grange JT, Corbett SW. Violence against emergency medical services personnel. Prehosp Emerg Care 2002;6:186-90.

3 Mock EF, Wrenn KD, Wright SW, et al. Prospective field study of violence in emergency medical services calls. Ann Emerg Med 1998;32:33-6.

4 Pollack KM, Agnew J, Slade MD, et al. Use of employer administrative databases to identify systematic causes of injury in aluminum manufacturing. Am J Ind Med 2007:50:676-86

5 Reichard AA, Marsh SM, Moore PH. Fatal and nonfatal injuries among emergency medical technicians and paramedics. Prehosp Emerg Care 2011;15:511-17.

6 Boal WL, Leiss JK, Ratcliffe JM, et al. The national study to prevent blood exposure in paramedics: rates of exposure to blood. Int Arch Occup Environ Health 2010;83:191-9.

7 Suyama J, Rittenberger JC, Patterson PD, et al. Comparison of public safety provider injury rates. Prehosp Emerg Care 2009;13:451-5.

8 Patterson PD, Arnold RM, Abebe K, et al. Variation in emergency medical technician partner familiarity. Health Serv Res 2011;46:1319-31.

9 Patterson PD, Weaver MD, Frank RC, et al. Association between poor sleep, fatigue, and safety outcomes in emergency medical services providers. Prehosp Emerg Care 2012;16:86-97. 
10 Williamson A, Lombardi DA, Folkard $\mathrm{S}$, et al. The link between fatigue and safety. Accid Anal Prev 2011;43:498-515.

11 Patterson PD, Weaver MD, Hostler $D$, et al. The shift length, fatigue, and safety conundrum in EMS. Prehosp Emerg Care 2012;16:572-6.

12 Occupational Safety and Health Administration. "Frequently Asked Questions: Extended Unusual Work Shifts". United States Department of Labor. www.osha. gov/OshDoc/data_Hurricane_Facts/faq_longhours.html (accessed 1 Jun 2014).

13 Wagstaff AS, Sigstad Lie JA. Shift and night work and long working hours-a systematic review of safety implications. Scand J Work Environ Health 2011:37:173-85.

14 Kirkwood S. Studies prove 24-hour shifts unsafe: another ethical dilemma for EMS leaders JEMS. San Diego, CA: PennWell Corporation, 2012.

15 NTSB. A review of flightcrew-involved major accidents of US air carriers, 1978 through 1990. Washington DC: National Transportation Safety Board, 1994.

16 Smith-Jentsch KA, Kraiger K, Cannon-Bowers JA, et al. Do familiar teammates request and accept more backup? Transactive memory in air traffic control. Hum Factors 2009:51:181-92.

17 Harrison DA, Mohammed S, McGrath JE, et al. Time matters in team performance: effects of member familiarity, entrainment, and task discontinuity on speed and quality. Personnel Psychol 2003;56:633-69.

18 Bledsoe BE, Porter RS, Cherry RA. Essentials of paramedic care: principles \& practice. Upper Saddle River, NJ: Pearson Prentice Hall, 2011.

19 Gershon RR, Vlahov D, Kelen G, et al. Review of accidents/injuries among emergency medical services workers in Baltimore, Maryland. Prehosp Disaster Med 1995;10:14-18.

20 Mock EF, Wrenn KD, Wright SW, et al. Anxiety levels in EMS providers: effects of violence and shifts schedules. Am J Emerg Med 1999;17:509-11.

21 Labor Force Statistics from the Current Population Survey. Labor Force Characteristics (CPS). Bureau of Labor Statistics, 2014.

22 Azaroff LS, Levenstein C, Wegman DH. Occupational injury and illness surveillance: conceptual filters explain underreporting. Am J Public Health 2002;92:1421-9.

23 Oleinick A, Gluck JV, Guire KE. Establishment size and risk of occupational injury. Am J Ind Med 1995:28:1-21.

24 Allen TL, Delbridge TR, Stevens MH, et al. Intubation success rates by air ambulance personnel during 12-versus 24-hour shifts: does fatigue make a difference? Prehosp Emerg Care 2001;5:340-3.

25 Thomas F, Hopkins RO, Handrahan DL, et al. Sleep and cognitive performance of flight nurses after 12-hour evening versus 18-hour shifts. Air Med J 2006;25:216-25.

26 Guyette FX, Morley JL, Weaver MD, et al. The effect of shift length on fatigue and cognitive performance in air medical providers. Prehosp Emerg Care 2013;17:23-8.
27 Maguire BJ, Smith S. Injuries and fatalities among emergency medical technicians and paramedics in the United States. Prehosp Disaster Med 2013;28:376-82.

28 Bender R, Blettner M. Calculating the "number needed to be exposed" with adjustment for confounding variables in epidemiological studies. J Clin Epidemiol 2002;55:525-30.

29 Cone DC, Brice JH, Delbridge TR, et al. Emergency medical services: clinical practice and systems oversight. Chichester, West Sussex: John Wiley \& Sons Inc., 2015.

30 Folkard S, Tucker P. Shift work, safety and productivity. Occup Med (Lond) 2003;53:95-101.

31 Lamond N, Dawson D. Quantifying the performance impairment associated with fatigue. J Sleep Res 1999;8:255-62.

32 Channouf $N$, L'Ecuyer $P$, Ingolfsson A, et al. The application of forecasting techniques to modeling emergency medical system calls in Calgary, Alberta. Health Care Manag Sci 2007;10:25-45.

33 Patterson PD, Moore CG, Weaver MD, et al. Mobile phone text messaging intervention to improve alertness and reduce sleepiness and fatigue during shiftwork among emergency medicine clinicians: study protocol for the SleepTrackTXT pilot randomized controlled trial. Trials 2014;15:244.

34 Frakes MA, Kelly JG. Sleep debt and outside employment patterns in helicopter air medical staff working 24-hour shifts. Air Med J 2007;26:45-9.

35 Takeyama $\mathrm{H}$, Itani T, Tachi N, et al. Effects of a modified ambulance night shift system on fatigue and physiological function among ambulance paramedics. J Occup Health 2009;51:204-9.

36 Patterson PD, Buysse DJ, Weaver MD, et al. Real-time fatigue reduction in emergency care clinicians: the SleepTrackTXT randomized trial. Am J Ind Med 2015. In press.

37 Probst TM, Estrada AX. Accident under-reporting among employees: testing the moderating influence of psychological safety climate and supervisor enforcement of safety practices. Accid Anal Prev 2010;42:1438-44.

38 Weaver MD, Wang HE, Fairbanks RJ, et al. The association between EMS workplace safety culture and safety outcomes. Prehosp Emerg Care 2012;16:43-52.

39 Stimpfel AW, Sloane DM, Aiken LH. The longer the shifts for hospital nurses, the higher the levels of burnout and patient dissatisfaction. Health Aff (Millwood) 2012;31:2501-9

40 Ruser JW. Examining evidence on whether BLS undercounts workplace injuries and illnesses. Mon Labor Rev 2008;131:20-32.

41 Tucker S, Diekrager D, Turner N, et al. Work-related injury underreporting among young workers: prevalence, gender differences, and explanations for underreporting. J Saf Res 2014;50:67-73.

42 Barger LK, Ayas NT, Cade BE, et al. Impact of extended-duration shifts on medical errors, adverse events, and attentional failures. PLoS Med 2006:3:e487. 